\title{
Multiband Hypersound Filtering in Two-Dimensional Colloidal Crystals: Adhesion, Resonances, and Periodicity
}

\author{
Bartlomiej Graczykowski,* Nicolas Vogel, Karina Bley, Hans-Jürgen Butt, and George Fytas
}

Cite This: Nano Lett. 2020, 20, 1883-1889

Read Online

ABSTRACT: The hypersonic phonon propagation in large-area twodimensional colloidal crystals is probed by spontaneous micro Brillouin light scattering. The dispersion relation of thermally populated Lamb waves reveals multiband filtering due to three distinct types of acoustic band gaps. We find Bragg gaps accompanied by two types of hybridization gaps in both sub- and superwavelength regimes resulting from contact-based resonances and nanoparticle eigenmodes, respectively. The operating $\mathrm{GHz}$ frequencies can be tuned by particle size and depend on the adhesion at the contact interfaces. The experimental dispersion relations are well represented by a finite element method model enabling identification of observed modes. The presented approach also allows for contactless study of the contact stiffness of submicrometer particles, which reveals size

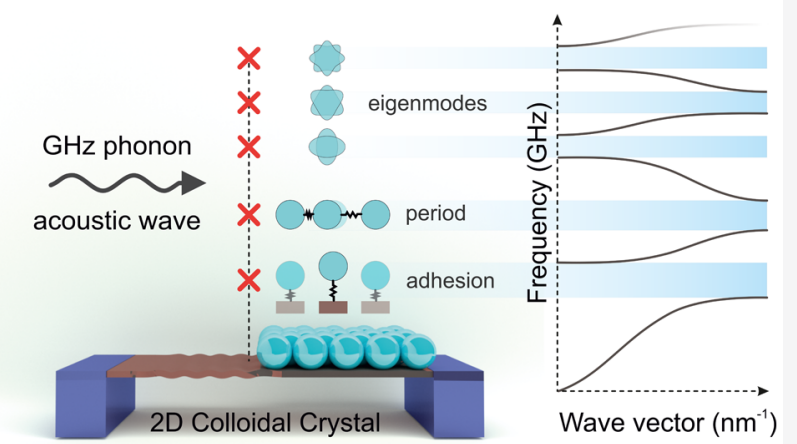
effect deviating from macroscopic predictions.

KEYWORDS: Colloidal crystals, phononics, acoustic band gap, Lamb waves, Brillouin light scattering, GHz signal filtering

$\mathrm{P}$ honons, quanta of acoustic field, similarly as photons and electrons, are carriers of energy, momentum, and information. High-frequency phonons in the $\mathrm{GHz}-\mathrm{THz}$ regime corresponding to submicrometer wavelengths are responsible for hypersound and heat transport, thus having importance for wireless communication, optomechanics, and thermal energy harvesting. ${ }^{1}$ Periodically structured materials that take advantage of the wave-like nature of phonons, such as phononic crystals (PnCs) and acoustic metamaterials, have been shown to enable new mechanical and acoustic features, including negative effective moduli and densities, frequency filtering, and acoustic cloaking. ${ }^{2-7}$ Recently, this field has experienced a tremendous growth of research in topological PnCs showing unidirectional propagation of sound immune to structural imperfections. ${ }^{8,9}$

To date, the majority of studies on PnCs and acoustic metamaterials has involved macroscopic structures aimed to affect $\mathrm{Hz}-\mathrm{kHz}$ acoustic waves/phonons. Nowadays, however, there is a significant need in developing PnCs in the nanoscale and operating in $\mathrm{GHz}$ as well as $\mathrm{THz}$ frequency regimes. ${ }^{6,10,11}$ Nevertheless, nanofabrication of hypersonic PnCs faces several practical challenges preventing their implementation in everyday devices. The patterning needs to be performed over large areas/volumes of matrices being CMOS compatible for easy integration without sophisticated instrumentation. Furthermore, fabricated materials should keep structural order and coherence of $\mathrm{GHz}$ acoustic signals over long length scales. Selfassembly of nanoparticles has emerged as a solution for lowcost mass production. ${ }^{12}$ Propagation of $\mathrm{GHz}$ phonons in three- dimensional (3D) self-assembled PnCs, typically known as colloidal crystals, composed of glass or polymer nanoparticles was initially investigated by means of Brillouin light scattering (BLS) ${ }^{13-17}$ More recently, contact-based modes of colloidal crystals have been extensively studied using the pump-probe technique. ${ }^{18-25}$ This approach, unlike BLS, is limited to sub$\mathrm{GHz}$ frequencies and often requires additional fabrication of a transducer for the acoustic wave generation.

Two-dimensional (2D) self-assembled PnCs, known as colloidal monolayers, ${ }^{12}$ offers a low-cost and large-area platform for harnessing high-frequency phonons. These materials, if supported by ultrathin membranes, can overcome the issue of signal/energy losses into the substrate. The use of inorganic membranes provides a mechanically robust framework serving as a waveguide for a discrete set of dispersive (Lamb) waves that can be tuned by the membrane thickness. $^{26,27}$ The monolayer-membrane architecture enables use of stiff and low-losses materials which can maintain signal coherence, adding functionality to $\mathrm{PnC}$. This solution goes hand in hand with new features of PnCs, that is, elastic and thermal size effects, unexplored schemes of band gap opening,

Received: December 11, 2019

Revised: January 29, 2020

Published: February 4, 2020 

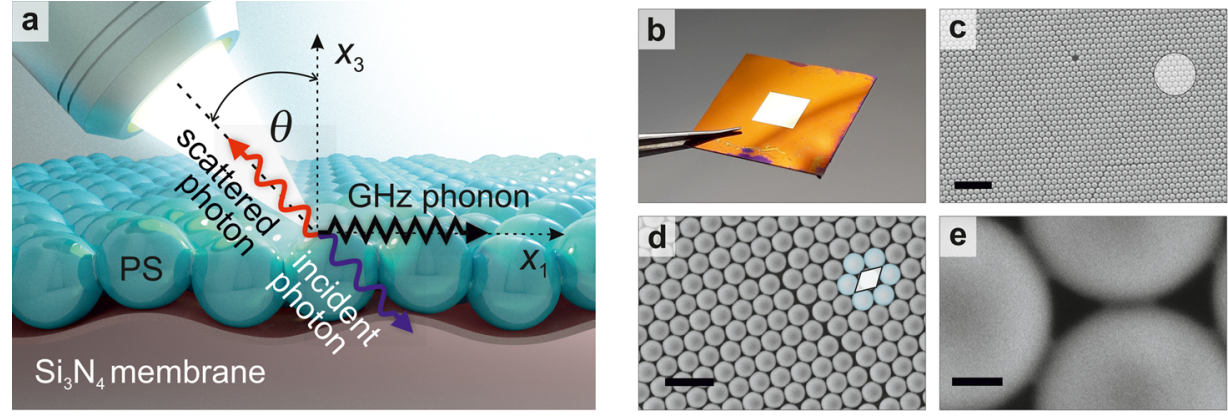

Figure 1. (a) Schematic illustration of the sample and the $\mu \mathrm{BLS}$ experiment. (b) Optical image of the sample. PS monolayer is deposited over the whole top surface of $\mathrm{Si}_{3} \mathrm{~N}_{4}$ chip, while the central bright $3 \times 3 \mathrm{~mm}^{2}$ square is fully suspended. Top view SEM images of samples with nanoparticle diameter (c) $d=333 \mathrm{~nm}$ and (d, e) $d=430 \mathrm{~nm}$. Dashed line lighter circle in (c) indicates approximately the area measured in the BLS experiment. In (d) light rhombus and cyan circles denote the lattice unit cell and the first nearest neighbors, respectively. Scale bars in (c), (d), and (e) are 2 $\mu \mathrm{m}, 1 \mu \mathrm{m}$ and $100 \mathrm{~nm}$, respectively.

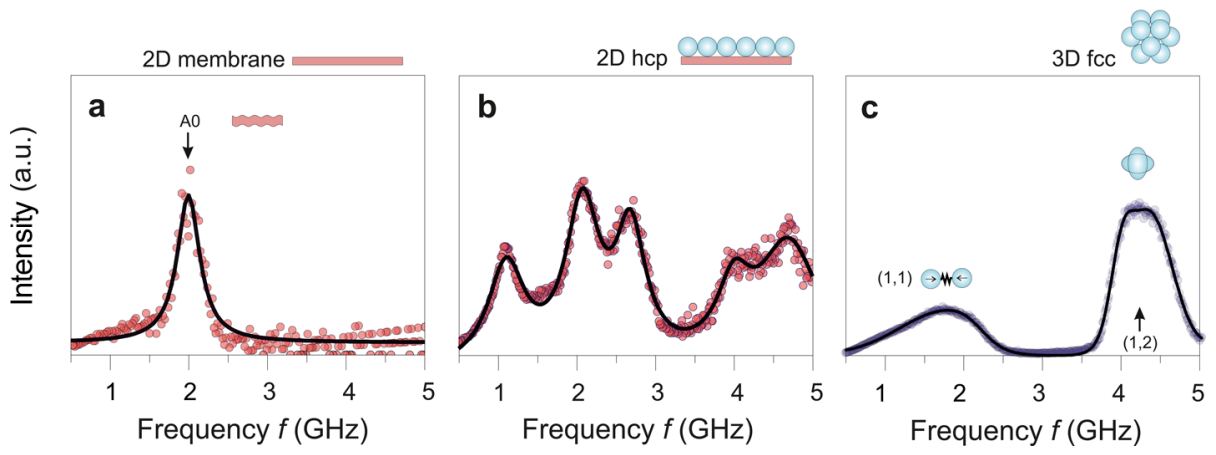

Figure 2. Measured (circles) normalized BLS spectra at $q=10 \mu \mathrm{m}^{-1}$ obtained for samples (a) A [membrane] and (b) B [ $\left.d=245 \mathrm{~nm}\right]$. (c) BLS spectrum of self-assembled 3D fcc cluster made of PS nanoparticles with $d=245 \mathrm{~nm}$. Solid lines correspond to Lorentzian multipeak fits of the experimental data.

as well as with experimental challenges related to their investigation and understanding in the high-frequency regime.

In this work, we study the phonon propagation in large-area 2D PnCs composed of spherical polystyrene (PS) nanoparticles self-assembled on an ultrathin $\mathrm{Si}_{3} \mathrm{~N}_{4}$ membrane (Figure 1). We use micro BLS $(\mu \mathrm{BLS})^{28,29}$ to record the dispersion of thermally populated $\mathrm{GHz}$ phonons. To interpret the experimental results, we develop a finite element method (FEM) model. We find significant modification of the phonon dispersion revealing three types of band gaps that are related to nanoparticle: membrane contact resonance (hybridization), lattice period (Bragg), and local resonances of nanoparticles (hybridization). The latter type, unlike for the vast majority of PnCs and metamaterials, appears above the Bragg band gap frequency, that is, in the superwavelength regime. ${ }^{2,3,30}$ All these mechanisms contribute to multiband filtering of hypersound that can be easily tailored by means of the nanoparticle size governing lattice spacing, frequencies of local resonances, and adhesion forces. Noteworthy, for the latter, we find a size effect and significant discrepancy from macroscopic predictions.

The samples were made of hexagonally close-packed (hcp) monolayers of PS nanoparticles deposited on $3 \times 3 \mathrm{~mm}^{2}$ and $t$ $=50 \mathrm{~nm}$-thick $\mathrm{Si}_{3} \mathrm{~N}_{4}$ membranes (Figure $1 \mathrm{~b}$ ). We used emulsion polymerization ${ }^{31}$ to prepare monodispersed dispersions of PS nanoparticles, which were self-assembled on the membranes using a pre-assembly at the air/water interface and subsequent transfer to the target membrane. ${ }^{32}$ We fabricated monolayer-membrane samples with nanoparticle diameters $d=$ $2 R=245,333$, and $430 \mathrm{~nm}$, which are labeled samples $\mathrm{B}, \mathrm{C}$, and $D$, respectively. A pristine membrane, labeled sample A, was used as a reference. In addition, we prepared three reference samples using PS nanoparticles of the same size as those of monolayer-membrane samples albeit assembled into $3 \mathrm{D}$ fcc clusters by means of drop cast evaporation. Such structures were extensively investigated in prior studies ${ }^{13,33}$ and here are utilized to highlight similarities and foremost differences with 2D hcp monolayer-membrane structures. Figure 1a-e shows a schematic view of the experiment, representative optical image, and SEM images from large to small length scales, respectively. A schematic side view, representative optical, and SEM images of the monolayermembrane samples are shown in Figure $1 \mathrm{a}-\mathrm{e}$ ), respectively. Typically, the in-plane translational symmetry extends over tens of micrometers.

We recorded the dispersion of acoustic waves propagating in the samples at room temperature by means of angle-resolved $\mu \mathrm{BLS}$ in $\mathrm{p}-\mathrm{p}(\mathrm{TM}-\mathrm{TM})$ backscattering geometry, as shown schematically in Figure la. The incident laser light at $\lambda_{0}=532$ $\mathrm{nm}$ was focused on the sample by means of $10 \times$ microscope objective with 0.25 numerical aperture. The scattered light was collected by the same objective and analyzed by a tandem type Fabry-Perot interferometer. The laser spot size on the sample and the corresponding power were in the order of a few micrometers and $<500 \mu \mathrm{W}$, respectively. BLS probes the frequency shift $f$ of the laser light inelastically scattered by thermally populated acoustic phonons. For a pristine membrane, which is well-approximated as a homogeneous elastic continuum, the scattering wave vector $\mathbf{q}$ is the same as the wave vector $\mathbf{k}$ of the acoustic phonons. However, for periodic structures the momentum conservation defines the 


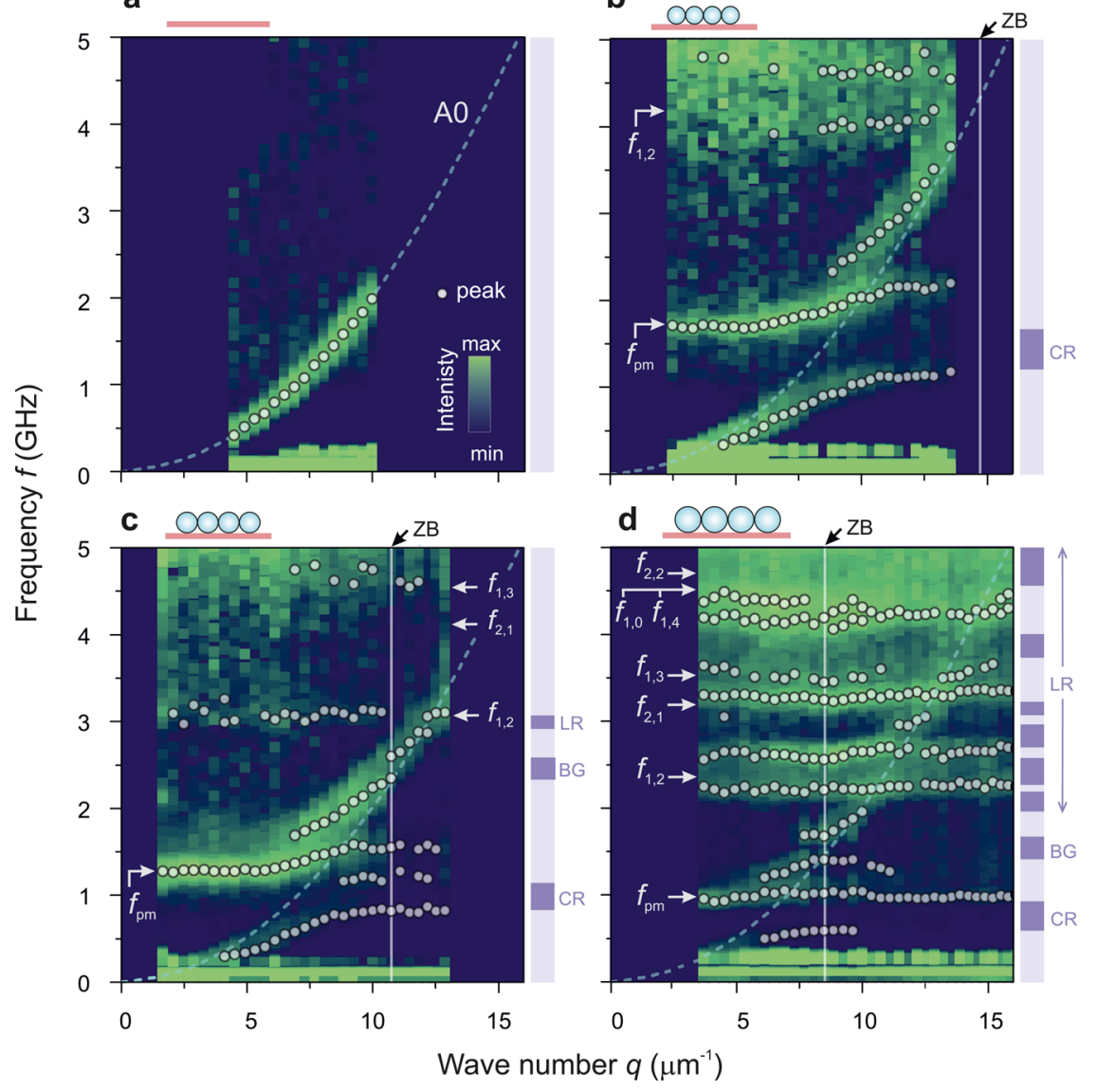

Figure 3. Experimental phonon dispersion relations $f(q)$ for (a) bare 50 nm-thick $\mathrm{Si}_{3} \mathrm{~N}_{4}$ membrane [sample A] and 50 nm membrane coated with a PS nanoparticle monolayer with diameters (b) $d=245 \mathrm{~nm}$ [sample B], (c) $d=333 \mathrm{~nm}$ [sample C], and (d) $d=430 \mathrm{~nm}$ [sample D]. Right-hand side bars denote band gaps (shading), where BG, CR, and LR stand for Bragg, nanoparticle-membrane contact resonance, and local resonance gap, respectively. Vertical solid lines denote the Brillouin zone boundary (ZB) at the M point. Dashed curves indicate the calculated dispersion of the fundamental antisymmetric Lamb mode (A0) of the bare membrane. Color scale indicates the BLS signal intensity normalized by the thermal phonon population factor. Circles stand for the peak position fitted by a Lorentzian function. Arrows indicate frequencies of particle-membrane contact resonance $\left(f_{\mathrm{pm}}\right)$ and unperturbed spheroidal Lamb modes $\left(f_{n, l}\right)$.

scattering wave vector as $\mathbf{q}=\mathbf{k} \pm \mathbf{G}$. In particular, for the hexagonal lattice $\mathbf{G}=(2 \pi /(a \sqrt{3}))\left[m_{1} \sqrt{3},-m_{1}+2 m_{2}\right]$ is a reciprocal lattice vector with lattice parameter $a$ and integers $m_{1}, m_{2}$. Following previous studies ${ }^{29}$ of thin membranes, we define the magnitude of the scattering wave vector $\mathbf{q}$ as $q=$ $4 \pi \sin \theta / \lambda_{0}$ and assume the superposition of photoelastic and moving-interface (surface ripple) effects contributing to BLS spectra. Figure 2a,b displays BLS spectra obtained for samples $\mathrm{A}$ and $\mathrm{B}$ at $q=10 \mu \mathrm{m}^{-1}$, which were normalized by the thermal population factor. ${ }^{34}$ The single peak present in the spectrum of the bare membrane can be assigned to a fundamental antisymmetric (flexural, A0) Lamb plate wave mode. ${ }^{26}$ PS monolayer on top of the membrane results in increased complexity of the spectrum, as evident in Figure 2b. However, the latter spectrum is distinct from that of the corresponding $3 \mathrm{D}$ control sample showed in Figure 2c. Two peaks in Figure $2 \mathrm{c}$ are attributed to the dipolar (low frequency) and quadrupolar (higher frequency) spheroidal Lamb modes of nanoparticles. ${ }^{14,33}$ In principle, the contribution of spheroidal Lamb modes to BLS is due to the photoelastic effect and depends on deformation field and dimensionless magnification factor $q R .{ }^{35}$ For a free homogeneous sphere, eigenfrequencies of Lamb modes are given by the formula $f_{n, l}=A_{n, l} v_{\mathrm{T}} / d$, where $n$ and $l$ are integers defining radial and angular dependence of the displacement, respectively, $v_{\mathrm{T}}$ is the transverse speed of sound in bulk PS, and $A_{n, l}$ is a dimensionless mode- and material-dependent parameter. ${ }^{33,36}$ The low-frequency peak in Figure $2 c$ originates in $(1,1)$ mode, which is nonzero as one would expect for a single sphere due to nanoparticlenanoparticle interactions. The peak line shape resembles phonon density of states (DOS) of propagating waves in the fcc cluster, as $q$ is ill-defined due to the light multiple scattering from the $3 \mathrm{D}$ cluster. ${ }^{14}$ The second peak is associated with $(1,2)$ mode, the expected frequency of which is indicated by an arrow in Figure 2c. The asymmetry of this peak is due to contact-induced splitting of $(1,2)$ mode into weakly dispersive branches. ${ }^{33}$ In principle, the BLS spectra of $3 \mathrm{D}$ clusters characterize spheroidal Lamb modes (torsional remain silent) and contain information about interparticle forces. By comparing Figure $2 b, c$, we notice that the asymmetric peak of $(1,2)$ mode in the 3D fcc cluster turns into two peaks in the 2D hcp monolayer-membrane. The difference between the spectra in Figure $2 b, c$ is even more evident in the low frequency range. The broad asymmetric peak of $(1,1)$ mode in the $3 \mathrm{D}$ sample is replaced by three distinct peaks recorded in the monolayer-membrane. A similar comparison of the BLS spectra of the other samples can be found in Supporting Information (SI). 
Figure 3 summarizes angle-resolved $\mu \mathrm{BLS}$ measurements for all membrane-monolayer samples as dispersion relations $f(q)$. Here, the color scale refers to the normalized intensity of the scattered light, and the circles stand for the frequencies at the peak positions. The dispersion relation of sample A displayed in Figure 3a shows only one branch that we identified as a flexural (A0) wave of a bare membrane. ${ }^{26}$ The fundamental symmetric (dilatational, SO) and shear-horizontal (SH0) modes are not BLS active due to zero (SHO) or small (SO) out-of-plane displacement $\left(u_{3}\right)$ for the measured range of wave numbers $q .{ }^{29}$ As follows from Figure $3 b-d$, the adhered monolayers in the samples B-D result in severely modified and complex dispersion relations compared to the dispersion of sample A.

At first glance, we can indicate three distinct effects and corresponding partial gaps related to the measured bands, that is, (i) Bragg reflections, hybridization of A0 mode with (ii) nanoparticle-membrane contact-based resonance, or (iii) spheroidal modes of nanoparticles. In each case, we define the band gaps for the modes of predominant out-of-plane displacement and indicate them only for the measured range of wave numbers $q$. For samples $C$ and $D$, we identify clear zone folding and Bragg band gaps (BG) at frequencies $f \approx 2.5 \mathrm{GHz}$ and $f \approx 1.5 \mathrm{GHz}$, respectively. This observation confirms the presence of wave-like/coherent effects and long-range order of PnCs extending over distances larger than the measured wavelengths. This phenomenon was neither observed nor modeled in previous works on similar, albeit sub-GHz, structures. Figure $3 c$,d suggests that the spectral position of BG can be estimated at the crossing of A0 mode and zone boundary (ZB). This provides simple geometric means for tunability of this type of a stop band, as $\mathrm{A} 0$ and $\mathrm{ZB}$ can be adjusted by the membrane thickness and nanoparticle diameter, respectively.

The horizontal branches that appear above $f \approx 2 \mathrm{GHz}$ in dispersion relations of samples B-D can be attributed to the spheroidal $(n, l)$ modes of PS nanoparticles. ${ }^{35,36}$ This assignment is supported by BLS spectra of the reference fcc clusters showing similarities with the accumulative spectra of the corresponding monolayer-membrane samples (SI, Figure S1). We use the calculated frequencies of spheroidal modes of free PS nanoparticles (arrows in Figure 3c,d) for a preliminary identification of these branches.

In Figure $3 \mathrm{~b}$,d, we observe a split of the $f_{1,2}$ mode that is represented by two distinct branches. A similar split was not captured for sample C, probably due to adverse $q R$ magnification factor and thereby weak scattering intensity for the $(1,2)$ mode. $^{35}$ The $(1,2)$ mode split can be explained by the presence of nanoparticle-nanoparticle and nanoparticlemembrane contacts. ${ }^{23,33}$ We will discuss this phenomenon in more detail later in this work, making use of FEM modeling. In the case of higher order $(n, l)$ modes, as those in the dispersion relation of sample $\mathrm{D}$, it cannot be clearly stated that the mode split is experimentally resolved. Although anticipated, it could be masked by a close neighborhood of branches as for instance those related to $(2,1)$ and $(1,3)$ modes. Despite this ambiguity, we observe a clear hybridized interaction of the spheroidal modes with the flexural wave at frequencies above $B G$, as evident in Figure $3 \mathrm{~d}$. This results in a unique feature of membrane-based PnCs, namely, opening of the local resonance band gaps (LR) in the superwavelength regime. This phenomenon was studied theoretically for pillared membranes in terms of the impact on heat transport at $\mathrm{THz}$ frequencies ${ }^{37}$ and, more recently, demonstrated experimentally in the $\mathrm{kHz}$ regime for a macroscopic trampoline metamaterial. ${ }^{38}$

Band diagrams of samples B-D reveal contact-based modes falling below BG that is in the subwavelength regime typical for acoustic metamaterials. ${ }^{3,5,39}$ These branches are a consequence of the hybridization and avoided crossing between A0 wave and the nanoparticle-membrane contact resonance. As we will further show using FEM, this resonance originates from the spheroidal $(1,1)$ mode. From Figures $3 b-d$ we can conclude that corresponding contact resonance (CR) stop bands appear in the hypersonic regime but at wavelengths longer than the lattice parameter. That type of a band gap was previously observed in the sub- $\mathrm{GHz}$ range for acoustic metamaterials $^{18,19,21,22,24}$ and modeled using Hertzian contact ${ }^{40,41}$ and van der Waals-type adhesive forces. ${ }^{2}$

The frequency of the nanoparticle-membrane resonance denoted as $f_{\mathrm{pm}}$ in Figure 3 yields information about the nanoparticle-membrane adhesion force. Assuming $q \rightarrow 0$ and negligible effect of nanoparticle-nanoparticle contacts, the normal contact stiffness $K_{\mathrm{N}}$ can be determined from the twomass oscillator equation $2 \pi f_{\mathrm{pm}}=\sqrt{K_{\mathrm{N}}\left(m_{\mathrm{p}}^{-1}+m_{\mathrm{m}}^{-1}\right)}$, where $m_{\mathrm{p}}$ and $m_{\mathrm{m}}$ denote masses of the PS nanoparticle and corresponding fraction of the membrane, respectively (Figure $4 a)$. Based in the latter expression, the contact resonance gap
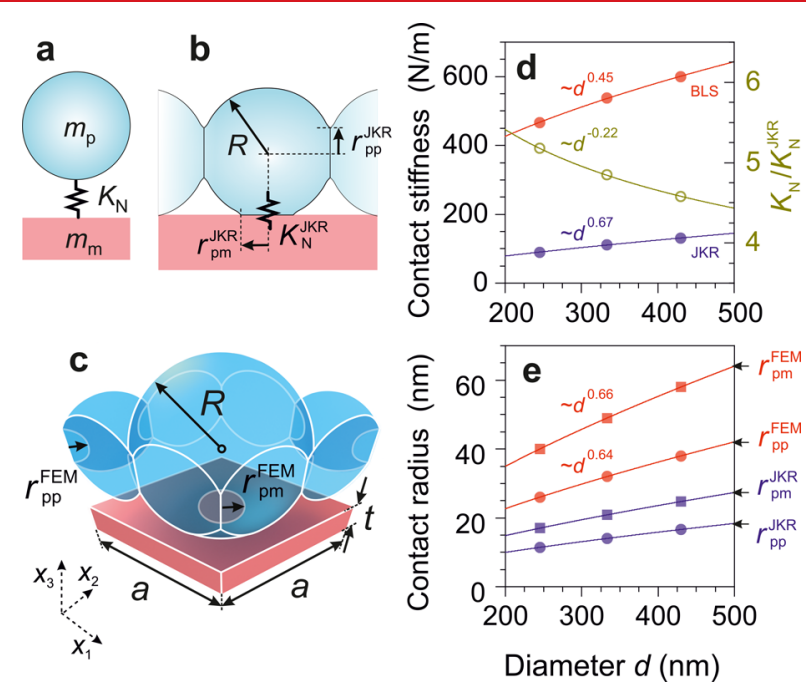

Figure 4. (a) Schematics of the nanoparticle-membrane contact stiffness $K_{\mathrm{N}}$ and (b) JKR model of nanoparticle-membrane and nanoparticle-nanoparticle contacts. (c) 3D scheme of FEM unit cell (see text). (d) Measured (BLS) and predicted (JKR) contact stiffness $K_{\mathrm{N}}$. (e) Fitted (FEM) from the experiment and predicted (JKR) contact radii of the nanoparticle-nanoparticle and nanoparticlemembrane interfaces.

depends on the nanoparticles mass, lattice packing, and strength of the nanoparticle-membrane bonding. The determined $K_{\mathrm{N}}$ is plotted in Figure $4 \mathrm{~d}$ and compared with expected values calculated by means of Johnson-KendallRoberts (JKR) model. ${ }^{43}$ For small displacements around the equilibrium point in JKR model, the linearized contact stiffness $K^{\mathrm{KRR}}$ (Figure $4 \mathrm{~b}$ ) is given by ${ }^{41,43,44}$

$$
K_{\mathrm{N}}^{\mathrm{JKR}}=\frac{9}{5}\left(\frac{3}{4} \pi W_{\mathrm{pm}} R^{2} E_{\mathrm{pm}}^{2}\right)^{1 / 3}
$$


a

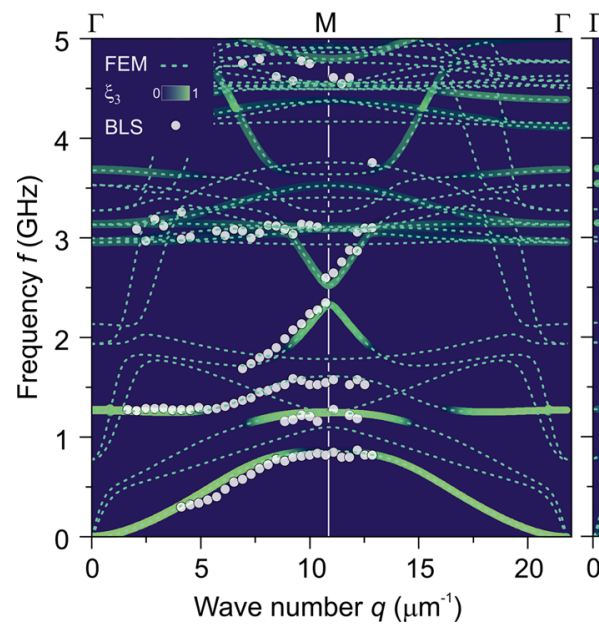

b

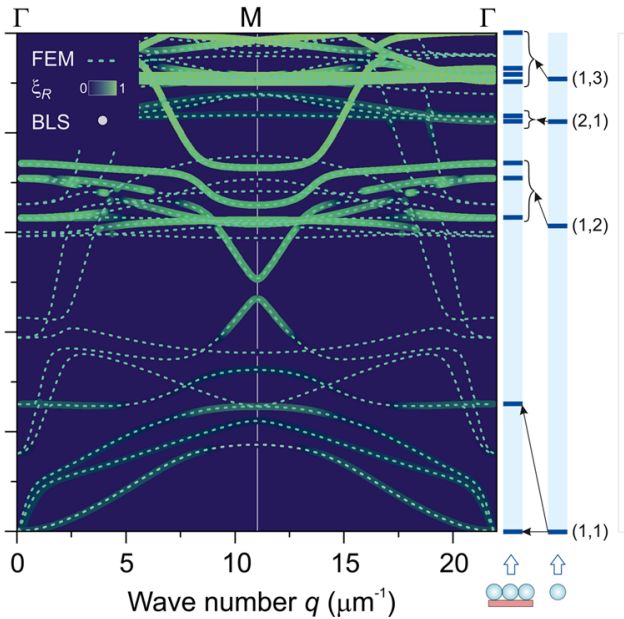

C

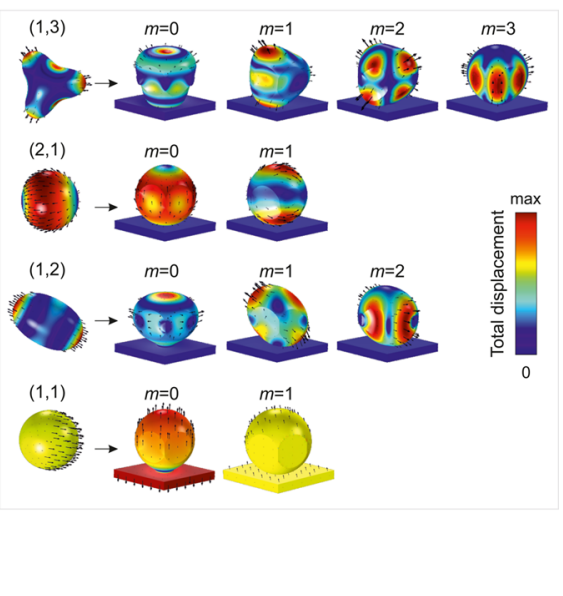

Figure 5. (a, b) Dispersion relations (dashed lines) calculated for sample $C[d=333 \mathrm{~nm}]$ with overlaid color intensity plots indicating (a) out-ofplane displacement $\left(\xi_{3}\right)$ and (b) radial displacements $\left(\xi_{R}\right)$. Circles in (a) stand for BLS experimental data. Right-hand panel of (b) schemes $2 l+1$ hybridization of BLS active spheroidal $(n, l)$ modes at $q=0$ due to nanoparticle-membrane and nanoparticle-nanoparticle contacts, where $m$ stands for the azimuthal number. Corresponding FEM displacement fields are displayed in (c).

where $E_{\mathrm{pm}}=5.91 \mathrm{GPa}$ is the nanoparticle-membrane effective modulus, $W_{\mathrm{pm}}=0.103 \mathrm{~J} / \mathrm{m}^{2}$ is the work of adhesion of PS$\mathrm{Si}_{3} \mathrm{~N}_{4}$, and $R$ is the nanoparticle radius. Both values are derived in SI (Section II). As we can infer from Figure $4 d$, for each sample, the measured contact stiffness $K_{\mathrm{N}}$ is about five times higher than what is predicted by JKR model. This deviation stems from additional physical bonding due to liquid or solid bridges resulting from impurities in the fabrication that are evident in SEM image in Figure 1e. ${ }^{25}$ Notably, the deviation from the model increases as the nanoparticle diameter becomes smaller. Conclusively, this manifests the impact of the real size of the contact interfaces on the phonon propagation in hypersonic colloidal PnCs in addition to the geometry and bulk elastic properties.

Theoretical models used in previous studies ${ }^{18,20,45}$ did not consider two phenomena evident in our measurements, namely, interaction of the propagating waves with local resonances and periodicity of the material. Therefore, to capture these features and explain $(1,2)$ mode split we developed a FEM model. Figure 4c schemes FEM 3D unit cell that includes lateral periodicity and assumes fused contacts with fully deformable spheres and membrane. PS spheres of radius $R$ are connected to each other and to a membrane of thickness $t$ by fully deformable circular interfaces with radii $r_{\mathrm{pp}}$ and $r_{\mathrm{pm}}$, respectively. The periodicity of the structure in the $x_{1} x_{2}$ plane is implemented by applying Bloch-Floquet periodic boundary conditions to all vertical walls of the unit cell. In the fitting procedure, we used two adjustable parameters, radii of nanoparticle-membrane $\left(r_{\mathrm{pm}}\right)$ and nanoparticle-nanoparticle $\left(r_{\mathrm{pp}}\right)$ contact areas. In addition to the dispersion relation, we used FEM to determine the contribution of surface ripple and photoelastic mechanisms to BLS by means of two parameters $\xi_{3}$ and $\xi_{R}$, respectively (SI, Section V). The superposition of these effects as well as size effects (membrane thickness, $q R$ factor) are beyond the scope of this work. Further details and material properties used in the FEM model can be found in the SI (Section V).

Figure 5a shows good agreement of the calculated dispersion relation with the experimental data obtained for sample $\mathrm{C}(d=$ $333 \mathrm{~nm}$ ). In addition, the experimental BLS intensity follows the calculated $\xi_{3}$ that is related to the magnitude of the BLS surface ripple mechanism. The discrepancies above about 2.5 $\mathrm{GHz}$ can result from the simplification, that is, neglected contribution of the photoelastic effect and its interference with the moving-interface effect, which can enhance as well as cancel the BLS signal. ${ }^{28,29}$ Nevertheless, FEM allows sorting of $(n, l)$ modes in terms of the sphere radial displacement. For that purpose we define the $\xi_{R}$ parameter, which resembles BLS activity of Lamb modes due to photoelastic effect (SI, Section $\mathrm{V}) .{ }^{35}$ We note that this approach does not include the effect of the $q R$ magnification factor. ${ }^{35}$ Figure $5 b$ shows the dispersion relation calculated for sample $\mathrm{C}$ with a color scale overlaid reflecting the magnitude of $\xi_{R}$. The frequency levels illustrate the split of $2 l+1$ degenerate vibrations of $(n, l)$ modes of a free sphere resulting from nanoparticle-membrane and nanoparticle-nanoparticle contacts and thereby symmetry lowering. $^{22,33,46}$ For transparency, we limit the analysis to BLS active modes at $q=0$. To identify the modes, we utilized FEM displacement fields showed in Figure $5 c$ which are sorted with respect to $(n, l)$ parent mode and the azimuthal number $m$. The example of $(1,2)$ mode at $q=0$ shows a split into two doublets $(m=1, m=2)$ and one singlet $(m=0)$, which we resolve as two peaks by means of BLS. The situation is much more complex for $q \neq 0$. Namely, the degeneracy is not present, and all five modes are of different frequencies. Furthermore, they interact with propagating waves in the membrane and their BLS activity varies with $q$. A similar analysis can be performed for all spheroidal modes undergoing mode split as schemed in Figure 5 b,c. Here, for instance, $(1,1) m=0$ mode with nanoparticle and membrane vibrating out-of-phase in the outof-plane direction is the nanoparticle-membrane contact resonance. The comparison of experimental and calculated dispersion relations for samples B and D can be found in SI (Section V).

The contact radii fitted by FEM and plotted in Figure 4e reveal a trend that follows the JKR model, ${ }^{33,41,42}$ namely $r_{\mathrm{pp}}^{\mathrm{FEM}}, r_{\mathrm{pm}}^{\mathrm{FEM}} \propto d^{2 / 3}$. In principle, the JKR model allows us to predict the contact radii from the formula (Figure $4 \mathrm{~b}$ ):

$$
r^{\mathrm{JKR}}=\left(6 \pi W R_{\mathrm{eff}}^{2} / E_{\mathrm{eff}}\right)^{1 / 3}
$$


where for the nanoparticle-membrane interface, the work of adhesion is $W=W_{\mathrm{pm}}=0.1036 \mathrm{~J} / \mathrm{m}^{2}$, effective radius is $R_{\text {eff }}=R$, and $E_{\mathrm{eff}}=E_{\mathrm{pm}}=5.91 \mathrm{GPa}$ is the effective moduli. In the case of the nanoparticle-nanoparticle contact in eq 2 , we substitute $W$ $=W_{\mathrm{pp}}=0.064 \mathrm{~J} / \mathrm{m}^{2}, R_{\mathrm{eff}}=R / 2$, and $E_{\mathrm{eff}}=E_{\mathrm{pp}}=3.01 \mathrm{GPa}($ see SI, Sections III and IV). From Figure $4 \mathrm{e}$, we can conclude that for both types of interfaces, the JKR model predicts over twice lower contact radii as compared to values fitted by FEM model for all samples. This goes along with the already mentioned underestimation of the contact stiffnesses by JKR model with respect to those measured by BLS. In principle, our results call into question the applicability of JKR model to assemblies of submicrometer particles with unavoidable fabrication imperfections.

In summary, we investigated the propagation of hypersonic phonons in large-area self-assembled 2D colloidal PnCs utilizing nondestructive $\mu \mathrm{BLS}$ technique. We showed that these materials can simultaneously host three distinct mechanisms for the phonon dispersion modification toward opening of stop bands for $\mathrm{GHz}$ acoustic waves. Each mechanism can be tailored by simple geometrical means as they result from (i) the lattice period, (ii) nanoparticlemembrane adhesion, and (iii) mechanical eigenmodes of nanoparticles. We found Bragg band gaps accompanied by subwavelength (contact resonance) and, noteworthy, multiple superwavelength (local resonance) gaps. We developed a finite element method model, which captured the physical phenomena with nanoparticle-nanoparticle and nanoparticle-membrane contact areas as fitting parameters. On the experimental side, we showed that $\mu \mathrm{BLS}$ provides a new means for contactless characterization of the interface mechanics at the submicrometer scale. We envision self-assembled 2D PnCs as a robust, chip-scale platform to study $\mathrm{GHz}$ signal processing for next-generation telecommunication devices, for tailoring sub-Kelvin thermal transport by coherent effects, ${ }^{47}$ for lightsound interaction in optomechanics and for nonlinear effects. ${ }^{45,48,49}$ These materials hold promise for downscaling topological acoustics to the hypersonic regime.

\section{ASSOCIATED CONTENT}

\section{SI Supporting Information}

The Supporting Information is available free of charge at https://pubs.acs.org/doi/10.1021/acs.nanolett.9b05101.

Material properties; acumulative BLS spectra; work of adhesion; effective contact elastic constant and radius; and finite element method model (PDF)

\section{AUTHOR INFORMATION}

\section{Corresponding Author}

Bartlomiej Graczykowski - Faculty of Physics, Adam Mickiewicz University, 61-614 Poznan, Poland; Max Planck Institute for Polymer Research, 55128 Mainz, Germany; ○ orcid.org/0000-0003-4787-8622; Email: graczykowski@ mpip-mainz.mpg.de

\section{Authors}

Nicolas Vogel - Institute of Particle Technology, FriedrichAlexander University Erlangen-Nürnberg, 91058 Erlangen, Germany; 이이이.org/0000-0002-9831-6905

Karina Bley - Institute of Particle Technology, FriedrichAlexander University Erlangen-Nürnberg, 91058 Erlangen, Germany
Hans-Jürgen Butt - Max Planck Institute for Polymer Research, 55128 Mainz, Germany; (i) orcid.org/0000-0001-5391-2618

George Fytas - Max Planck Institute for Polymer Research, 55128 Mainz, Germany

Complete contact information is available at: https://pubs.acs.org/10.1021/acs.nanolett.9b05101

\section{Notes}

The authors declare no competing financial interest.

\section{ACKNOWLEDGMENTS}

B.G. acknowledges the support from the Foundation for Polish Science (POIR.04.04.00-00-5D1B/18), Polish National Science Centre (UMO-2018/31/D/ST3/03882). The work was supported by the ERC AdG SmartPhon (grant no. 694977).

\section{REFERENCES}

(1) Volz, S.; Ordonez-Miranda, J.; Shchepetov, A.; Prunnila, M.; Ahopelto, J.; Pezeril, T.; Vaudel, G.; Gusev, V.; Ruello, P.; Weig, E. M.; et al. Nanophononics: state of the art and perspectives. Eur. Phys. J. B 2016, 89, 15.

(2) Hussein, M. I.; Leamy, M. J.; Ruzzene, M. Dynamics of phononic materials and structures. Historical origins, recent progress, and future outlook. Appl. Mech. Rev. 2014, 66, No. 040802.

(3) Deymier, P. A. Acoustic metamaterials and phononic crystals; Springer Science+Business Media: New York, 2013; Vol. 173.

(4) Laude, V. Phononic crystals: artificial crystals for sonic, acoustic, and elastic waves; Walter de Gruyter GmbH \& Co KG: Berlin, 2015; Vol. 26.

(5) Khelif, A.; Adibi, A. Phononic Crystals; Springer Science +Business Media: New York, 2015.

(6) Maldovan, M. Phonon wave interference and thermal bandgap materials. Nat. Mater. 2015, 14, 667.

(7) Graczykowski, B.; Sledzinska, M.; Alzina, F.; Gomis-Bresco, J.; Reparaz, J.; Wagner, M.; Torres, C. S. Phonon dispersion in hypersonic two-dimensional phononic crystal membranes. Phys. Rev. B: Condens. Matter Mater. Phys. 2015, 91, No. 075414.

(8) Mousavi, S. H.; Khanikaev, A. B.; Wang, Z. Topologically protected elastic waves in phononic metamaterials. Nat. Commun. 2015, 6, 8682.

(9) Wang, P.; Lu, L.; Bertoldi, K. Topological Phononic Crystals with One-Way Elastic Edge Waves. Phys. Rev. Lett. 2015, 115, 104302.

(10) Benchabane, S.; Reinhardt, A. Fundamentals and Applications of Acoustic Metamaterials; John Wiley \& Sons, Ltd: Hoboken, NJ, 2019; Chapter 8, pp 207-262.

(11) Yazdani, N.; Jansen, M.; Bozyigit, D.; Lin, W. M.; Volk, S.; Yarema, O.; Yarema, M.; Juranyi, F.; Huber, S. D.; Wood, V. Nanocrystal superlattices as phonon-engineered solids and acoustic metamaterials. Nat. Commun. 2019, 10, 4236.

(12) Vogel, N.; Retsch, M.; Fustin, C.-A.; del Campo, A.; Jonas, U. Advances in colloidal assembly: the design of structure and hierarchy in two and three dimensions. Chem. Rev. 2015, 115, 6265-6311.

(13) Cheng, W.; Wang, J.; Jonas, U.; Fytas, G.; Stefanou, N. Observation and tuning of hypersonic bandgaps in colloidal crystals. Nat. Mater. 2006, 5, 830.

(14) Mattarelli, M.; Montagna, M.; Still, T.; Schneider, D.; Fytas, G. Vibration spectroscopy of weakly interacting mesoscopic colloids. Soft Matter 2012, 8, 4235-4243.

(15) Akimov, A. V.; Tanaka, Y.; Pevtsov, A. B.; Kaplan, S. F.; Golubev, V. G.; Tamura, S.; Yakovlev, D. R.; Bayer, M. Hypersonic Modulation of Light in Three-Dimensional Photonic and Phononic Band-Gap Materials. Phys. Rev. Lett. 2008, 101, No. 033902.

(16) Wu, S.; Zhu, G.; Zhang, J. S.; Banerjee, D.; Bass, J. D.; Ling, C.; Yano, K. Anisotropic lattice expansion of three-dimensional colloidal crystals and its impact on hypersonic phonon band gaps. Phys. Chem. Chem. Phys. 2014, 16, 8921-8926. 
(17) Still, T.; Cheng, W.; Retsch, M.; Sainidou, R.; Wang, J.; Jonas, U.; Stefanou, N.; Fytas, G. Simultaneous Occurrence of StructureDirected and Particle-Resonance-Induced Phononic Gaps in Colloidal Films. Phys. Rev. Lett. 2008, 100, 194301.

(18) Boechler, N.; Eliason, J. K.; Kumar, A.; Maznev, A. A.; Nelson, K. A.; Fang, N. Interaction of a Contact Resonance of Microspheres with Surface Acoustic Waves. Phys. Rev. Lett. 2013, 111, No. 036103. (19) Khanolkar, A.; Wallen, S.; Abi Ghanem, M.; Jenks, J.; Vogel, N.; Boechler, N. A self-assembled metamaterial for Lamb waves. Appl. Phys. Lett. 2015, 107, No. 071903.

(20) Wallen, S. P.; Maznev, A. A.; Boechler, N. Dynamics of a monolayer of microspheres on an elastic substrate. Phys. Rev. B: Condens. Matter Mater. Phys. 2015, 92, 174303.

(21) Hiraiwa, M.; Abi Ghanem, M.; Wallen, S. P.; Khanolkar, A.; Maznev, A. A.; Boechler, N. Complex Contact-Based Dynamics of Microsphere Monolayers Revealed by Resonant Attenuation of Surface Acoustic Waves. Phys. Rev. Lett. 2016, 116, 198001.

(22) Vega-Flick, A.; Duncan, R.; Wallen, S.; Boechler, N.; Stelling, C.; Retsch, M.; Alvarado-Gil, J.; Nelson, K.; Maznev, A. Vibrational dynamics of a two-dimensional microgranular crystal. Phys. Rev. B: Condens. Matter Mater. Phys. 2017, 96, No. 024303.

(23) Vega-Flick, A.; Duncan, R.; Wallen, S.; Boechler, N.; Stelling, C.; Retsch, M.; Alvarado-Gil, J.; Nelson, K.; Maznev, A. Contactbased and spheroidal vibrational modes of a hexagonal monolayer of microspheres on a substrate. Wave Motion 2018, 76, 122-133.

(24) Otsuka, P. H.; Mezil, S.; Matsuda, O.; Tomoda, M.; Maznev, A. A.; Gan, T.; Fang, N.; Boechler, N.; Gusev, V. E.; Wright, O. B. Timedomain imaging of gigahertz surface waves on an acoustic metamaterial. New J. Phys. 2018, 20, No. 013026.

(25) Abi Ghanem, M.; Khanolkar, A.; Wallen, S. P.; Helwig, M.; Hiraiwa, M.; Maznev, A. A.; Vogel, N.; Boechler, N. Longitudinal eigenvibration of multilayer colloidal crystals and the effect of nanoscale contact bridges. Nanoscale 2019, 11, 5655.

(26) Lamb, H. On waves in an elastic plate. Proc. R. Soc. London, Ser. A 1917, 93, 114-128.

(27) Graczykowski, B.; Gomis-Bresco, J.; Alzina, F.; Reparaz, J.; Shchepetov, A.; Prunnila, M.; Ahopelto, J.; Torres, C. S. Acoustic phonon propagation in ultra-thin $\mathrm{Si}$ membranes under biaxial stress field. New J. Phys. 2014, 16, No. 073024.

(28) Graczykowski, B.; Sledzinska, M.; Placidi, M.; Saleta Reig, D.; Kasprzak, M.; Alzina, F.; Sotomayor Torres, C. Elastic Properties of Few Nanometers Thick Polycrystalline MoS2Membranes: A Nondestructive Study. Nano Lett. 2017, 17, 7647-7651.

(29) Graczykowski, B.; Gueddida, A.; Djafari-Rouhani, B.; Butt, H.J.; Fytas, G. Brillouin light scattering under one-dimensional confinement: Symmetry and interference self-canceling. Phys. Rev. B: Condens. Matter Mater. Phys. 2019, 99, 165431.

(30) Liu, Z.; Zhang, X.; Mao, Y.; Zhu, Y.; Yang, Z.; Chan, C. T.; Sheng, P. Locally resonant sonic materials. Science 2000, 289, 17341736.

(31) Qiu, D.; Cosgrove, T.; Howe, A. M. Narrowly Distributed Surfactant-Free Polystyrene Latex with a Water-Soluble Comonomer. Macromol. Chem. Phys. 2005, 206, 2233-2238.

(32) Vogel, N.; Goerres, S.; Landfester, K.; Weiss, C. K. A Convenient Method to Produce Close-and Non-close-Packed Monolayers using Direct Assembly at the Air-Water Interface and Subsequent Plasma-Induced Size Reduction. Macromol. Chem. Phys. 2011, 212, 1719-1734.

(33) Kim, H.; Cang, Y.; Kang, E.; Graczykowski, B.; Secchi, M.; Montagna, M.; Priestley, R. D.; Furst, E. M.; Fytas, G. Direct observation of polymer surface mobility via nanoparticle vibrations. Nat. Commun. 2018, 9, 2918.

(34) YU, P.; Cardona, M. Fundamentals of Semiconductors: Physics and Materials Properties, 4th ed.; Graduate Texts in Physics; SpringerVerlag: Berlin, 2010.

(35) Still, T.; Mattarelli, M.; Kiefer, D.; Fytas, G.; Montagna, M. Eigenvibrations of submicrometer colloidal spheres. J. Phys. Chem. Lett. 2010, 1, 2440-2444.
(36) Lamb, H. On the Vibrations of an Elastic Sphere. Proceedings of the London Mathematical Society 1881, s1-13, 189-212.

(37) Davis, B. L.; Hussein, M. I. Nanophononic metamaterial: Thermal conductivity reduction by local resonance. Phys. Rev. Lett. 2014, 112, No. 055505

(38) Bilal, O. R.; Foehr, A.; Daraio, C. Observation of trampoline phenomena in 3D-printed metamaterial plates. Extreme Mechanics Letters 2017, 15, 103-107.

(39) Maznev, A. A.; Gu, G.; yuan Sun, S.; Xu, J.; Shen, Y.; Fang, N.; yi Zhang, S. Extraordinary focusing of sound above a soda can array without time reversal. New J. Phys. 2015, 17, No. 042001.

(40) Hertz, H. On the contact of elastic solids. J. reine angew. Math 1881, 92, 156.

(41) Johnson, K. L. Contact mechanics; Cambridge university press: Cambridge, UK, 1987.

(42) Israelachvili, J. Intermolecular and Surface Forces; Elsevier Science: Amsterdam, 2015.

(43) Johnson, K. L.; Kendall, K.; Roberts, A. Surface energy and the contact of elastic solids. Proceedings of the royal society of London. A. mathematical and physical sciences 1971, 324, 301-313.

(44) Saeedi Vahdat, A.; Azizi, S.; Cetinkaya, C. Nonlinear dynamics of adhesive micro-spherical particles on vibrating substrates. J. Adhes. Sci. Technol. 2013, 27, 1712-1726.

(45) Wallen, S.; Lee, J.; Mei, D.; Chong, C.; Kevrekidis, P.; Boechler, N. Discrete breathers in a mass-in-mass chain with Hertzian local resonators. Phys. Rev. E: Stat. Phys., Plasmas, Fluids, Relat. Interdiscip. Top. 2017, 95, No. 022904.

(46) Girard, A.; Gehan, H.; Mermet, A.; Bonnet, C.; Lermé, J.; Berthelot, A.; Cottancin, E.; Crut, A.; Margueritat, J. Acoustic mode hybridization in a single dimer of gold nanoparticles. Nano Lett. 2018, $18,3800-3806$.

(47) Zen, N.; Puurtinen, T. A.; Isotalo, T. J.; Chaudhuri, S.; Maasilta, I. J. Engineering thermal conductance using a twodimensional phononic crystal. Nat. Commun. 2014, 5, 3435.

(48) Bunyan, J.; Vakakis, A. F.; Tawfick, S. Mechanisms for impulsive energy dissipation and small-scale effects in microgranular media. Phys. Rev. E 2015, 92, No. 062206.

(49) Wallen, S. P.; Boechler, N. Shear to longitudinal mode conversion via second harmonic generation in a two-dimensional microscale granular crystal. Wave Motion 2017, 68, 22-30. 